\title{
Prevenção do consumo de álcool em manuais escolares de Ciências Naturais portugueses
}

\author{
Augusta Diasi \\ Agrupamento de Escolas de Cabeceiras de Basto, Portugal \\ José Preciosoii \\ Universidade do Minho, Portugal
}

\begin{abstract}
Resumo
O consumo de álcool é um problema grave, muito prevalente e em crescimento entre adolescentes escolarizados. Por este motivo, a prevenção do consumo de álcool deve ser realizada na escola. Os manuais escolares são um recurso a ser usado nos processos preventivos. A presente investigação tem como objetivo analisar as abordagens ao tema do consumo de álcool propostas pelos manuais escolares portugueses de Ciências Naturais para o $9 .^{\circ}$ ano de escolaridade. Neste sentido, procedeu-se à análise de conteúdo de sete manuais escolares existentes em Portugal para o ano letivo de 2008/2009, que estarão em vigor até ao ano de 2014. Os ma-nuais escolares abordam a problemática do consumo de álcool de forma muito incompleta e superficial. Em nenhum manual são sugeridas atividades que desenvolvam nos estudantes competências para resistir às pressões que frequentemente conduzem ao consumo. Os autores dos manuais devem ter em conta a necessidade de aprofundar este tema.
\end{abstract}

Palavras-chave

Manuais escolares; Prevenção do alcoolismo; Educação; Adolescência

\section{Introdução1}

O consumo de álcool é responsável por 3,7\% do total de mortes e por 14,4\% da morbilidade a nível mundial (Organização Mundial de Saúde [OMS], 
2007). Em 2002 morreram 600.000 europeus de causas relacionadas com o alcoolismo, representando $6,3 \%$ de mortes prematuras, correspondendo 63.000 dessas mortes a jovens entre os 15 e os 29 anos de idade (OMS, 2005).

Embora o alcoolismo nos adultos seja um problema de saúde, social e económico grave e muito prevalente, o consumo abusivo de álcool pelos adolescentes e jovens tem consequências diferentes das evidenciadas nos adultos, devido à fase de desenvolvimento em que se encontram (Pechansky, Szobot, \& Scivoletto, 2004). O padrão de consumo de álcool dos jovens atuais é diferente dos do passado, caracterizando-se por um consumo compulsivo normalmente ao fim de semana. Este comportamento origina frequentemente problemas imediatos como a embriaguez, podendo levar ao coma, ou até mesmo à morte (Calafat \& Munar, 1999). O consumo excessivo e agudo de álcool pelos adolescentes e jovens adultos (Pechansky et al., 2004) tem sido a principal causa de acidentes de tráfego (Calafat, 2002). Em Portugal, os acidentes rodoviários são a principal causa de morte entre os jovens de 15 a 19 anos e estão entre as primeiras causas de morte entre os menores de 25 anos (Nogueira, Silva, Santos, \& Azevedo, 2008).

O consumo abusivo de álcool pelos jovens está também associado a comportamentos de risco, entre os quais se encontram as relações sexuais desprotegidas (Calafat, 2002), expondo-se ao contágio de doenças sexualmente transmissíveis como a SIDA, sífilis e aumentando o risco de gravidez indesejada (Calafat \& Munar, 1999). O consumo de álcool interfere ainda com os resultados escolares, uma vez que afeta a memória (McKinnon, O'Rourke, Thompson, \& Berumen, 2004).

Não obstante as consequências do consumo de álcool para os jovens, o relatório do European School Survey Project on Alcohol and Other Drugs (ESPAD) de 2011 (Hibell et al., 2012), que envolveu mais de 100.000 estudantes de 36 países europeus com idades compreendidas entre os $15 \mathrm{e}$ os 16 anos de idade, revelou que, em média, $56 \%$ dos rapazes e $50 \%$ das raparigas referiram ter consumido bebidas alcoólicas nos últimos 30 dias (que antecederam o inquérito do ESPAD). Mais de metade dos jovens de ambos os sexos tinham um consumo regular de álcool. Apesar de se registar uma prevalência elevada de consumidores de álcool entre adolescentes portugueses, a prevalência média registada no ano de 2011 (52\%) é mais 
baixa do que a prevalência média registada nos países participantes no ESPAD (56\%) (Hibell et al., 2012).

No que concerne ao consumo excessivo de álcool (5 ou mais bebidas na mesma ocasião) nos últimos 30 dias, é de $27 \%$ nos rapazes e $19 \%$ nas raparigas. Apesar de se registar uma prevalência elevada de "binge drinking" entre adolescentes portugueses, a prevalência média registada no ano de 2011 (22\%) é mais baixa do que a prevalência média registada nos países participantes no ESPAD (41\%). Comparativamente com o ano de 2007, registou-se um decréscimo apreciável de "binge drinking" em ambos os sexos (de $58 \%$ para $27 \%$ no sexo masculino e de $53 \%$ para $19 \%$ no sexo feminino). Apesar disto, a evolução do consumo nos últimos 16 anos parece apontar para uma tendência crescente desta forma de consumo, principalmente no sexo feminino (Hibell et al., 1997, 2000, 2004, 2009, 2012).

Os resultados do Health Behaviour in School Aged Children (HBSC) obtidos numa amostra total de mais de 207.000 estudantes, de 38 países e regiões da Europa e América do Norte, demonstram que, em Portugal, no ano de 2010, as bebidas mais consumidas semanalmente por rapazes de 15 anos de idade foram a cerveja ( $8 \%)$, seguindo-se as bebidas brancas $(5 \%)$, os alcopops (4\%) e o vinho (3\%). No que respeita às raparigas, do mesmo grupo etário, as bebidas preferencialmente consumidas semanalmente foram as bebidas brancas (4\%), seguindo-se a cerveja (3\%), os alcopops $(2 \%)$ e o vinho (1\%). Comparativamente com a média dos restantes países participantes no HBSC, Portugal regista prevalências mais baixas de consumo semanal para todos os tipos de bebidas e em ambos os sexos. No sexo masculino, as bebidas mais consumidas semanalmente acompanham as tendências europeias, sendo que a bebida mais consumida foi a cerveja (18\%), seguindo-se, com a mesma prevalência, as bebidas brancas e os alcopops ( $9 \%$ cada) e o consumo de vinho (6\%). Esta tendência não se regista no sexo feminino, já que a bebida mais consumida pela média de jovens participantes no estudo HBSC foram os alcopops (8\%), seguindo-se a cerveja (7\%), as bebidas brancas (6\%) e o vinho (4\%) (Currie et al., 2012).

\section{Prevenção do consumo de álcool}

Os comportamentos têm consequências sobre a saúde, pelo que a adoção de condutas adequadas, de modo a promover e a conservar a saúde, 
pode conseguir-se através da Educação para a Saúde e, particularmente, através da educação sobre o álcool e outras drogas (Calafat \& Munar, 1999). De forma a atingir as metas definidas, a OMS (2000) aponta como uma das medidas a inclusão, no sistema educativo, desde o pré-escolar, de formação alcoológica, implementada através de um programa integrado de formação, que desenvolva a resistência ao consumo precoce de álcool e a capacidade de efetuar escolhas saudáveis, estando previsto financiamento de atividades de educação para a saúde com verbas resultantes da cobrança de impostos da venda das bebidas alcoólicas.

Neste contexto, a redução do consumo de álcool apresenta-se como um dos objetivos primordiais da Organização Mundial de Saúde (OMS) e dos governos europeus (Pascual, 2002) e pode conseguir-se através da Educação para a Saúde e, particularmente, através da educação sobre o álcool e outras drogas, conforme anteriormente referido. O Ministério da Educação português definiu alguns temas transversais às diversas áreas disciplinares, um dos quais é a educação para a saúde e o bem-estar, do qual fazem parte a educação alimentar, a educação sexual e a educação para a prevenção de situações de risco pessoal, onde se inclui a prevenção do consumo de drogas (Ministério da Educação, 2001). Neste sentido, sendo objetivo prioritário da política educativa a adoção de medidas de promoção da saúde escolar, o Despacho interno de 27 de setembro de 2006 do Secretário de Estado da Educação determinou que os Agrupamentos/Escolas devem incluir no seu Projeto Educativo temáticas no âmbito da Promoção e Educação para a Saúde, sendo uma das mais importantes o consumo de substâncias psicoativas.

Em Portugal, o Grupo de Trabalho de Educação Sexual/Saúde (GTES) reforça a relevância e a necessidade da obrigatoriedade da Promoção e Educação para a Saúde nos estabelecimentos de ensino, do $1 .^{\circ}$ ao $12 .^{\circ}$ ano, fazendo parte integrante do Projeto Educativo de cada escola, com as devidas adaptações à realidade escolar, contemplando quatro áreas fundamentais de ação educativa: "Alimentação e atividade física; Consumo de substâncias psicoativas, tabaco, álcool e drogas; Sexualidade e infeções sexualmente transmissíveis, com relevância para a prevenção da Síndrome da Imunodeficiência Adquirida (SIDA); Violência em meio escolar/Saúde mental" (Sampaio, Baptista, Matos, \& Silva, 2007, p. 4). O mesmo grupo elaborou 
referenciais para os temas "Educação alimentar em meio escolar", "Consumo de substâncias psicoativas e prevenção em meio escolar" e "Prevenção da violência em meio escolar" (Sampaio et al., 2007, p. 5). É importante salientar que, não obstante a transversalidade da Educação e Promoção da Saúde, a disciplina de Ciências Naturais, em particular, dada a sua natureza, deve contribuir para a sua concretização em meio escolar e de forma mais relevante no $9 .^{\circ}$ ano de escolaridade, dado as orientações curriculares assim o indicarem. Desta forma, esta importantíssima missão faz parte das atribuições dos docentes de Ciências Naturais, pelo que os mesmos devem, na sua formação inicial, desenvolver competências que lhes permitam desempenhar, com sucesso, essa função.

\section{Os manuais escolares no ensino e na aprendizagem das Ciências}

Os investigadores e os especialistas na Educação em Ciências consideram que os manuais escolares são o recurso pedagógico que suporta o processo de planificação e ensino das ciências. Na realidade, na maior parte das salas de aula, os manuais escolares são utilizados e seguidos como se do currículo nacional se tratasse e determinam o que é ensinado e aprendido sobre a Ciência (Chiappetta, Fillman, \& Sethna, 1991). Efetivamente, os dados da investigação têm vindo a confirmar que o processo de ensino e aprendizagem é mediado pelos manuais escolares, sendo o recurso didático mais utilizado na sala de aula (Parcerisa, 1997).

Estudos efetuados em Portugal e Espanha sobre manuais escolares no primeiro ciclo do Ensino Básico demonstram que os docentes são muito influenciados pelas propostas de atividades apresentadas pelos manuais escolares, por vezes mais do que pelas orientações curriculares do Ministério da Educação (Parcerisa, 1997; Teixeira, Couceiro, Veiga, \& Martins, 1999). Salienta-se que o conhecimento científico chega a ser confundido com a informação contida nos manuais escolares, existindo a crença de que tudo o que um manual escolar contém é correto e adequado, quer em termos científicos, quer em termos didáticos (Teixeira et al., 1999). 


\section{Os manuais escolares e a prevenção do consumo de álcool}

De acordo com Gérard e Roegiers (1998), "Os manuais escolares de Ciências Naturais, do $9 .^{\circ}$ ano de escolaridade, têm função importante na Promoção e Educação para a Saúde e em particular na prevenção do consumo de álcool, devendo desenvolver nos alunos a capacidade de reflexão, ajudando-os a fazer opções adequadas e promovendo atitudes de rejeição do álcool" (p. 83).

Atendendo ao facto de as orientações curriculares de Ciências Naturais do $3 .^{\circ}$ Ciclo do Ensino Básico (português) proporem que a abordagem da prevenção do consumo de álcool seja feita no $9 .^{\circ}$ ano de escolaridade, integrado no tema "Viver melhor na Terra", na subunidade "Opções que interferem no equilíbrio do organismo", torna-se imperioso investigar os tópicos sobre o consumo de álcool presentes nos manuais do 9 . $^{\circ}$ ano e as atividades propostas para a sua abordagem. Assim, esta investigação tem como principal objetivo analisar as propostas de abordagem da temática da prevenção do consumo de álcool apresentadas pelos manuais escolares. Esta análise é importante pelo facto de a Lei $n .^{\circ} 47 / 2006$, de 28 de agosto, prever que os manuais escolares tenham um período de vigência, em regra, de seis anos, o que faz com que a revisão dos manuais em estudo esteja prevista para o ano letivo de 2013/2014, podendo os resultados desta investigação ajudar os autores a incorporar algumas sugestões.

\section{Metodologia}

\section{População e amostra}

Selecionamos para este estudo os sete manuais escolares do $9 .^{\circ}$ ano de escolaridade, de Ciências Naturais, das diversas editoras, apresentados para adoção em 2008 (Quadro 1).

Todos os manuais escolares utilizados no estudo têm como ano de edição 2008 e, de acordo com a Lei n. ${ }^{\circ}$ 47/2006, de 28 de agosto e o Despacho n. ${ }^{\circ}$ 29865/2007, de 30 de novembro, manter-se-ão em vigor durante seis anos (do ano letivo 2008/2009 ao ano letivo 2013/2014). 


\section{Quadro 1 - Código atribuído aos manuais escolares}

\begin{tabular}{cl}
\hline CÓDIGO & MANUAL ESCOLAR \\
\hline M1 & Silva, A., Santos, M., Gramaxo, F., Mesquita, A., Baldaia, L., \& Félix, J. (2008). Planeta \\
& vivo. Porto: Porto Editora. \\
M2 & Motta, L., \& Viana, M. (2008). Bioterra. Porto: Porto Editora. \\
M3 & Barros, A., \& Delgado, F. (2008). Planeta Terra. Carnaxide: Santillana Constância. \\
M4 & Antunes, C., Bispo, M., \& Guindeira, P. (2008). Descobrir a Terra 9. Porto: Areal Editores. \\
M5 & Deus, H., \& Albuquerque. F. (2008). Geovida. Lisboa: Lisboa Editora. \\
M6 & Campos, C., \& Delgado, Z. (2008). 9CN. Lisboa: Texto Editores. \\
M7 & Franco, N., Franco, E., \& Borges, M. (2008). Bios. Porto: Edições Asa. \\
\hline
\end{tabular}

O número total de páginas varia entre 192 e 231, tendo uma média de 213,4 páginas. No que respeita ao número de páginas onde a temática da prevenção do consumo de álcool é abordada, varia entre uma página e meia e quatro páginas, ou seja, com uma média de 2,7 páginas (Quadro 2).

\section{Quadro 2 - Caracterização da amostra de manuais escolares}

\begin{tabular}{|c|c|c|c|c|c|c|c|c|c|}
\hline \multicolumn{2}{|c|}{ Manuais Escolares } & M1 & M2 & M3 & M4 & M5 & M6 & M7 & \multirow{2}{*}{ Média } \\
\hline Ano de & dição & 2008 & 2008 & 2008 & 2008 & 2008 & 2008 & 2008 & \\
\hline \multirow{3}{*}{$\begin{array}{l}\mathrm{N}^{\circ} \\
\text { páginas }\end{array}$} & Total & 192 & 192 & 224 & 224 & 208 & 231 & 223 & 213,4 \\
\hline & $\begin{array}{c}\text { Sobre a } \\
\text { Prevenção } \\
\text { Consumo } \\
\text { Álcool }\end{array}$ & 4 & 2,5 & 1,5 & 2 & 3 & 2 & 4 & 2,7 \\
\hline & $\begin{array}{c}\% \text { Pre- } \\
\text { venção } \\
\text { Consumo } \\
\text { Álcool }\end{array}$ & $2,1 \%$ & $1,3 \%$ & $0,7 \%$ & $0,9 \%$ & $1,4 \%$ & $0,9 \%$ & $1,8 \%$ & $1,3 \%$ \\
\hline
\end{tabular}

\section{Seleção da técnica de recolha de dados}

A análise de documentos foi considerada a técnica adequada de recolha de dados. Esta é uma técnica que tem um enorme campo de aplicação, de acordo com Quivy e Campenhoudt (2003), e também se mostra adequada quando a análise incide sobre manuais escolares (Bardin, 1995).

\section{Instrumentos de recolha de dados: Elaboração e validação}

O objetivo deste estudo foi analisar as propostas (estratégias) de abordagem da temática da prevenção do consumo de álcool apresentadas 
nos manuais escolares de Ciências Naturais do $9 .^{\circ}$ ano de escolaridade. Para o efeito, construiu-se uma grelha de análise onde constavam as dimensões que se pretendiam medir (estando estas relacionadas com os objetivos do estudo) e as respetivas categorias.

$\mathrm{Na}$ elaboração da grelha de análise das estratégias preventivas sugeridas pelos manuais escolares foi utilizada a classificação para os modelos preventivos apresentada por Calafat e Munar (1999), que divide as intervenções preventivas do consumo de drogas, em geral, e do consumo de álcool, em particular, em: i) Informativas; ii) Afetivas-Centradas nas Pessoas/Baseadas nos valores e tomadas de decisões; iii) Centradas nas Situações/Competências Sociais. As primeiras caracterizam-se pela transmissão de informação de forma unidirecional, com pouca interatividade, e têm como principal finalidade a transmissão de informação sobre bebidas alcoólicas e as consequências do consumo. As intervenções baseadas "nos valores e tomada de decisões" têm por base o esclarecimento de valores, o auxílio dos jovens na definição das suas próprias prioridades e a orientação para a tomada de decisões adequadas. As intervenções que incidem sobre a promoção de competências sociais integram componentes como o treino de assertividade e de resolução de problemas, promovendo a capacidade crítica quanto à publicidade e à pressão dos colegas, sendo igualmente importante a promoção da resiliência.

Com base nas dimensões propostas por Calafat e Munar (1999) definiram-se, a priori, as categorias (Bardin, 1995). No entanto, segundo De Ketele e Roegiers (1999), o instrumento de recolha de informação é um utensílio aberto, não podendo ser totalmente definido, pelo que à medida que a recolha da informação foi sendo efetuada surgiu a necessidade de redefinir algumas categorias. As dimensões e as categorias de análise finais constam no Quadro 3.

O processo de validação destas grelhas passou pela apreciação por parte de especialistas, que verificaram a pertinência da informação a recolher, a sua validade interna e externa. Entre esses especialistas estavam dois docentes de didática das ciências, com muita experiência em investigação em Educação para a Saúde, e dois professores do Ensino Secundário. 


\section{Recolha de dados}

Neste estudo, a recolha de dados efetuou-se em três etapas: i) começou-se por analisar todos os manuais escolares e dossiês suplementares, identificando, em cada um deles, as páginas que abordavam a temática da prevenção do consumo de bebidas alcoólicas, com o objetivo de selecionar o conteúdo a ser analisado; ii) efetuou-se a análise de conteúdo dos manuais escolares (Bardin, 1995), sendo feita a identificação das estratégias e a sua inclusão em cada uma das dimensões e respetiva categoria de análise, pela primeira autora do estudo; iii) numa outra etapa, e para garantir a qualidade dos dados, a primeira autora do estudo repetiu a análise utilizando a mesma grelha. As discrepâncias observadas foram alvo de uma análise cuidadosa de forma a minimizar os erros inerentes à subjectividade da investigadora. A decisão final sobre a inclusão da estratégia na categoria ficou a cargo de um investigador sénior (o orientador do trabalho) após análise desses casos duvidosos com a primeira autora do estudo. Com este procedimento minimizou-se os erros inerentes à subjetividade que esta tarefa de classificação contém em si mesma.

\section{Resultados/conclusões}

A análise de conteúdo dos manuais escolares incidiu sobre as estratégias preventivas por eles utilizadas, procurando verificar se as mesmas se enquadram em estratégias "Informativas", "Afetivas - Centradas nas Pessoas" ou "Centradas nas Situações".

Observando o Quadro 3, constata-se que todos os manuais escolares de Ciências Naturais do $9 .^{\circ}$ ano apresentam conhecimentos sobre o consumo de álcool. 
Quadro 3 - Estratégias preventivas propostas pelos manuais escolares de Ciências Naturais do $9 .^{\circ}$ ano de escolaridade sobre a problemática do consumo do álcool

\begin{tabular}{|c|c|c|c|c|c|c|c|c|c|c|c|}
\hline & & \multicolumn{7}{|c|}{ MANUAIS ESCOLARES } & \multicolumn{3}{|c|}{ N. ${ }^{\circ}$ TOTAL } \\
\hline & ESTRATÉGIAS PREVENTIVAS & M1 & M2 & M3 & $\overline{M 4}$ & M5 & M6 & M7 & $\mathrm{s}$ & \pm & $\mathrm{N}$ \\
\hline \multirow{4}{*}{ 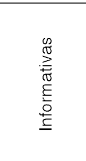 } & $\begin{array}{l}\text { Apresenta conhecimentos sobre o consumo de } \\
\text { álcool }\end{array}$ & $\mathrm{s}$ & s & $\mathrm{s}$ & $\mathrm{s}$ & $\mathrm{s}$ & $\mathrm{s}$ & $\mathrm{s}$ & 7 & 0 & 0 \\
\hline & Combate crenças sobre o consumo de álcool & $\mathrm{N}$ & \pm & $\mathrm{N}$ & $\mathrm{N}$ & $\mathrm{N}$ & $\mathrm{N}$ & \pm & 0 & 2 & 5 \\
\hline & $\begin{array}{l}\text { Ajuda a conhecer os níveis de consumo de álcool } \\
\text { de alto e baixo risco }\end{array}$ & \pm & \pm & N & $\mathrm{N}$ & $\mathrm{N}$ & $\mathrm{N}$ & \pm & 0 & 3 & 4 \\
\hline & $\begin{array}{l}\text { Ajuda a estabelecer niveis de consumo de álcool } \\
\text { aceitáveis e de risco }\end{array}$ & $\mathrm{N}$ & $\mathrm{N}$ & $\mathrm{N}$ & $\mathrm{N}$ & $\mathrm{N}$ & $\mathrm{N}$ & N & 0 & 0 & 7 \\
\hline \multirow{4}{*}{ 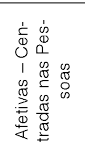 } & $\begin{array}{l}\text { Ajuda a refletir sobre os determinantes do consumo } \\
\text { do álcool }\end{array}$ & N & $\mathrm{N}$ & N & \pm & \pm & $\mathrm{N}$ & \pm & 0 & 3 & 4 \\
\hline & $\begin{array}{l}\text { Responsabiliza o indivíduo pelos próprios consu- } \\
\text { mos }\end{array}$ & $\mathrm{N}$ & $\mathrm{N}$ & $\mathrm{N}$ & $\mathrm{N}$ & $\mathrm{N}$ & $\mathrm{N}$ & \pm & 0 & 1 & 6 \\
\hline & Desenvolve competências de rejeição do álcool & $\mathrm{N}$ & $\mathrm{N}$ & $\mathrm{N}$ & $\mathrm{N}$ & $\mathrm{N}$ & $\mathrm{N}$ & \pm & 0 & 1 & 6 \\
\hline & Capacita para a tomada de decisões & $\mathrm{N}$ & $\mathrm{N}$ & $\mathrm{N}$ & $\mathrm{N}$ & $\mathrm{N}$ & $\mathrm{N}$ & \pm & 0 & 1 & 6 \\
\hline \multirow{4}{*}{ 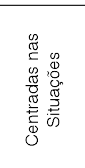 } & $\begin{array}{l}\text { Prepara os jovens para enfrentar situações de } \\
\text { oferta }\end{array}$ & $\mathrm{N}$ & $\mathrm{N}$ & N & $\mathrm{N}$ & $\mathrm{N}$ & $\mathrm{N}$ & N & 0 & 0 & 7 \\
\hline & $\begin{array}{l}\text { Prepara os jovens para enfrentar as pressǒes de } \\
\text { grupo }\end{array}$ & N & N & N & N & $\mathrm{N}$ & N & N & 0 & 0 & 7 \\
\hline & $\begin{array}{l}\text { Desenvolve a capacidade de prever as consequên- } \\
\text { cias das suas decisões }\end{array}$ & \pm & \pm & \pm & \pm & \pm & \pm & \pm & 0 & 7 & 0 \\
\hline & Treina a assertividade & $\mathrm{N}$ & $\mathrm{N}$ & $\mathrm{N}$ & $\mathrm{N}$ & $\mathrm{N}$ & $\mathrm{N}$ & $\mathrm{N}$ & 0 & 0 & 7 \\
\hline \multirow{3}{*}{ 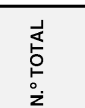 } & Estratégias preventivas utilizadas (S) & 1 & 1 & 1 & 1 & 1 & 1 & 1 & & & \\
\hline & $\begin{array}{l}\text { Estratégias preventivas utilizadas mas pouco de- } \\
\text { senvolvidas }( \pm)\end{array}$ & 2 & 3 & 1 & 2 & 2 & 1 & 7 & & & \\
\hline & Estratégias preventivas não utilizadas $(\mathrm{N})$ & 9 & 8 & 10 & 9 & 9 & 10 & 4 & & & \\
\hline
\end{tabular}

No que se refere à estratégia preventiva baseada no combate de crenças sobre o consumo de álcool, os manuais escolares M1, M3, M4, M5 e M6 não a utilizam, pois não fazem qualquer referência às crenças e mitos que se encontram associados ao consumo de álcool e, como tal, também não os desmistificam. Os manuais escolares M2 e M7 fazem uma breve abordagem à desmistificação de algumas crenças. Como se constata pela figura 1 , 0 manual escolar M2 procura romper a associação que existe entre as saídas de grupos de amigos, o divertimento e o consumo de álcool. 


\section{Figura 1 - Ilustração de uma atividade do manual escolar M2 (p. 14) relativamente à desmistificação da relação entre o convívio e o consumo de álcool}

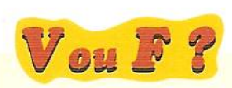
"As saídas em grupo só são
divertidas se toda a gente se
embebedar."

FALSO A capacidade de as pessoas se divertirem tem a ver com o convívio que se estabelece, os laços que se criam, o contexto em que os encontros acontecem e não com 0 consumo de substâncias psicoactivas.

O manual escolar M7 faz referência a mitos partilhados por inúmeras pessoas, como sejam "o álcool dá força, aquece e faz esquecer os problemas" (M7, 2008, p. 192), e propõe alguma reflexão, por exemplo, sobre a publicidade que, por vezes, passa uma imagem de glamour e sucesso social associado ao consumo de álcool. O mesmo manual escolar possui uma atividade intitulada "Consumo de substâncias e sua interferência na saúde: mitos e realidades", onde são apresentadas algumas afirmações para o aluno refletir, propondo-lhe que identifique os mitos e as realidades, apresentando frases como: "A ingestão de álcool não cria dependência; beber pouco não afecta as capacidades de condução" (M7, 2008, p. 174).

Conhecer os níveis de consumo de álcool de alto e baixo risco é outra estratégia preventiva que Calafat e Munar (1999) enquadram nas estratégias informativas. Os manuais escolares M3, M4, M5 e M6 não facultam ao aluno essa informação. O manual escolar M1, com a imagem da figura 2, permite que se faça a dedução que beber um copo de vinho diariamente é um consumo de baixo risco, mas, no entanto, o consumo num só dia de um elevado número de bebidas alcoólicas terá como consequência a embriaguez 
146 Augusta Dias \& José Precioso

e, se este facto se consumar diariamente, estaremos perante uma situação de alcoolismo crónico, tratando-se, por isso, de um consumo de alto risco.

Figura 2 - Ilustração do manual escolar M1 (p. 146) distinguindo alcoolismo agudo de alcoolismo crónico

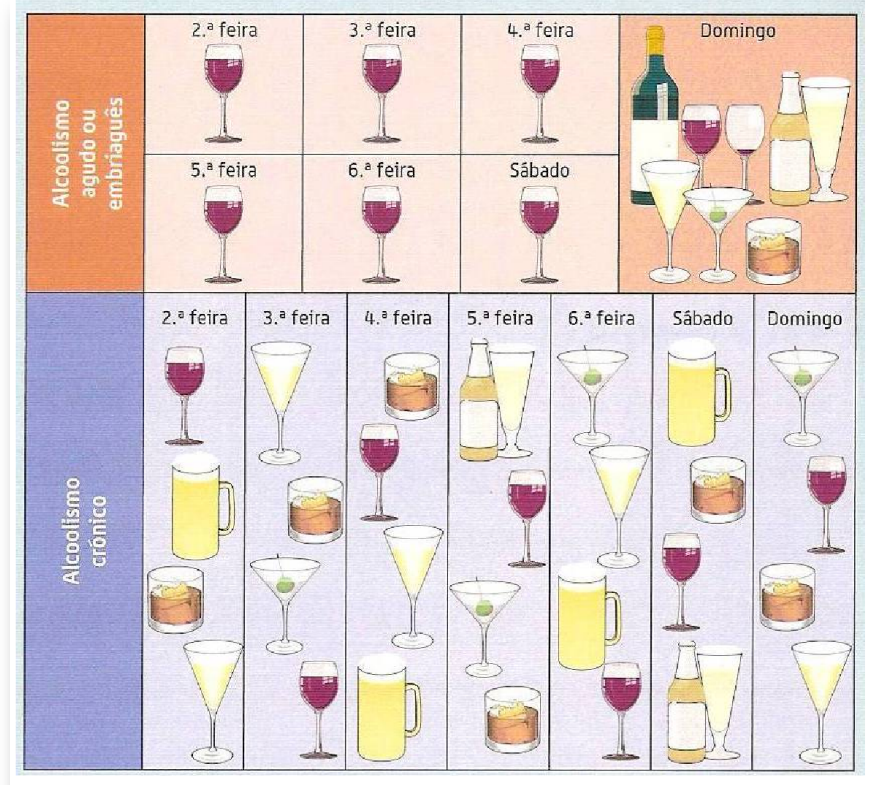

O manual escolar $\mathrm{M} 2$, com a imagem da figura 3, não permite conhecer efetivamente os níveis de consumo de álcool de alto e baixo risco. 
Figura 3 - llustração de uma atividade do manual escolar M2 (p. 146) relacionando as perturbações de comportamento com a Taxa de Alcoolemia no Sangue

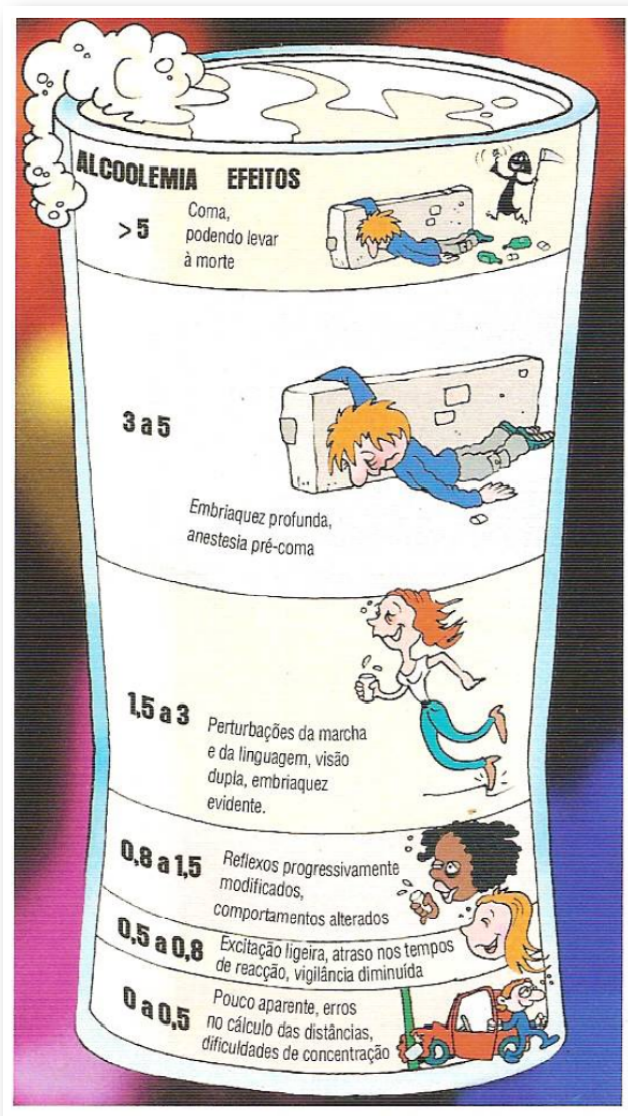

O manual escolar M7 refere, ainda que muito vagamente, que "Factores como idade, sexo, constituição física do indivíduo e hábitos de vida poderão condicionar a quantidade de álcool que uma pessoa pode ingerir sem perder, ainda que aparentemente, os reflexos e a coordenação motora" (M7, 2008, p. 194). 
No que se refere à estratégia de estabelecimento dos níveis de consumo de álcool aceitáveis e de risco, nenhum manual escolar propõe ao aluno qualquer atividade que o incentive a estabelecer esses níveis.

Ao analisar os manuais escolares relativamente às estratégias "Afetivas - Centradas nas Pessoas", procurou verificar-se se os mesmos contribuem para uma reflexão sobre os determinantes do consumo do álcool. No entanto, constata-se que os manuais escolares não apresentam momentos que permitam ao aluno refletir sobre os determinantes do consumo de álcool.

No que respeita à responsabilização dos alunos pelos próprios consumos, ao desenvolvimento de competências de rejeição do álcool e à capacitação dos alunos para a tomada de decisões, verifica-se que os manuais escolares apresentam imagens e atividades (figura 4 e figura 5) que o docente pode explorar para desenvolver essas competências nos alunos; no entanto, essa proposta de abordagem não é apresentada pelo manual escolar.

O manual escolar M7 propõe que se reflita sobre a "influência que a publicidade tem nos hábitos de consumo de álcool, alertando os alunos para a necessidade de espírito crítico enquanto consumidores", sugerindo também que seja explorada "a questão do falso sucesso (profissional, social) associado ao álcool" (M7, 2008, p. 192) (figura 6). Com esta proposta pode, de alguma forma, conseguir-se uma desmistificação de crenças associadas ao consumo de álcool e estimular o espírito crítico dos alunos, responsabilizando-os pelos próprios consumos, ao mesmo tempo que se podem desenvolver competências de rejeição do álcool, capacitando-os para a tomada de decisões. 
Figura 4 - llustração de uma atividade do manual escolar M1 (p. 147) estabelecendo a relação entre o alcoolismo e os acidentes rodoviários

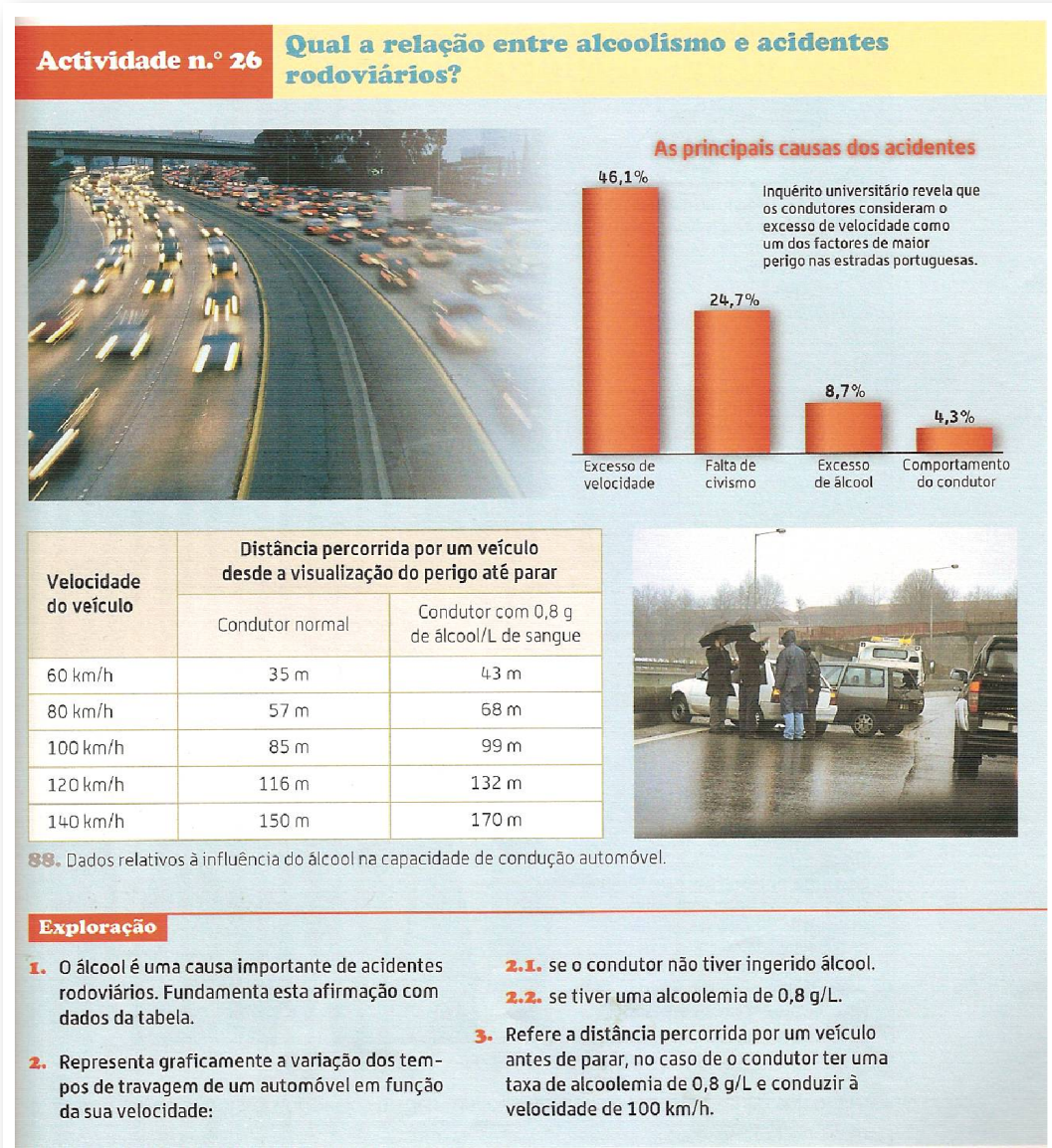


Figura 5 - llustração de uma atividade do manual escolar M5 (p. 125) relativa ao consumo de álcool entre os jovens

Actividade 2 O que vou descobrir.... analisando

\section{Consumos de álcool entre jovens}

Analisa os diferentes gráficos, que apresentam os consumos dos jovens (rapazes e raparigas) em função do tipo de bebida.

1. Caracteriza os comportamentos dos rapazes e das raparigas em função do tipo de bebida.

2. Refere qual o tipo de bebida que é consumido com mais regularidade por rapazes e raparigas.

3. Formula uma hipótese que explique a escolha referida na alínea anterior.

4. Prevê a evolução dos hábitos de consumo de álcool destes jovens.

Consumo de bebidas alcoolicas entre os jovens

Valores em percentagem referentes uo ono de 1998

Fonte: Observatório ao consumo de substáncias licitas e
illcitas em estudantes do ensino superior em Portugal.

Ropares Raparigos
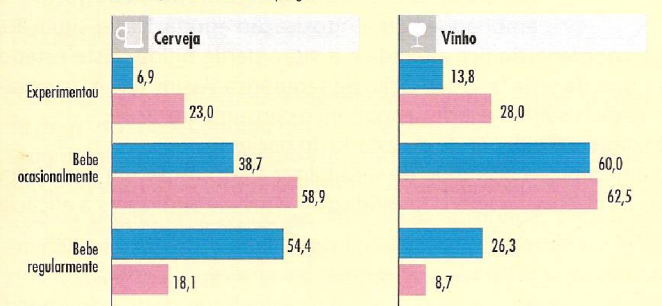

Whisky

10.7
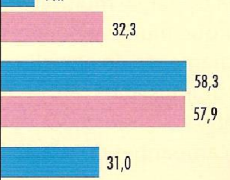

Figura $8>$ Consumo de bebidas alcoólicas entre os jovens portugueses.

9,8

Figura 6 - llustração de uma imagem do manual escolar M7 (p. 192) mostrando que a publicidade associa muitas vezes o glamour e sucesso social ao consumo de álcool

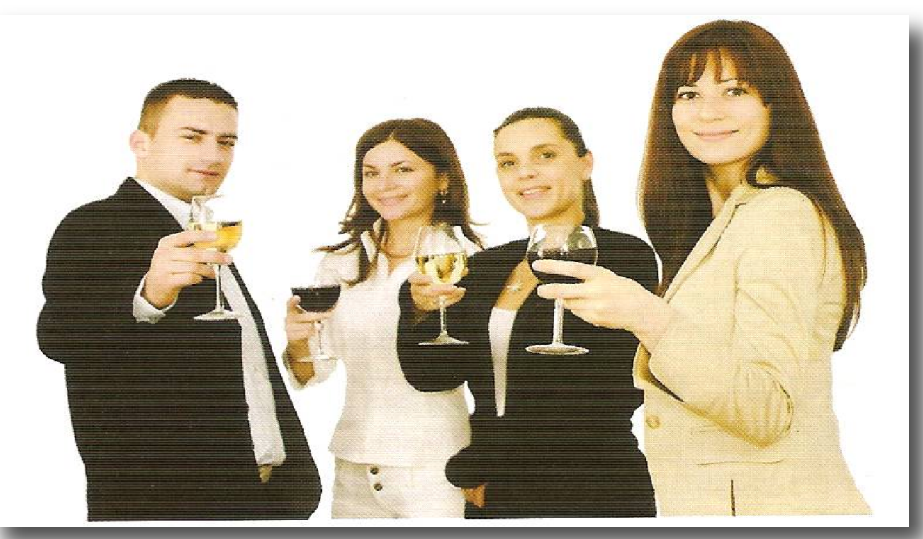


Seguidamente procurou verificar-se se os manuais escolares apresentam situações que preparem os alunos para rejeitar ofertas de consumo de álcool e lhes desenvolvam competências para enfrentar as pressões de grupo. Constatou-se que nenhum dos manuais escolares apresenta estas propostas de abordagem.

O manual escolar M6 apresenta a imagem da figura 7. No entanto, não propõe que a mesma seja explorada no sentido de desmistificar a relação entre divertimento e consumo de álcool, bem como a resistência à pressão social e do grupo para esse consumo.

Figura 7 - Ilustração de uma imagem do manual escolar M6 (p. 209) apresentando um grupo de jovens a conviver e a consumir álcool

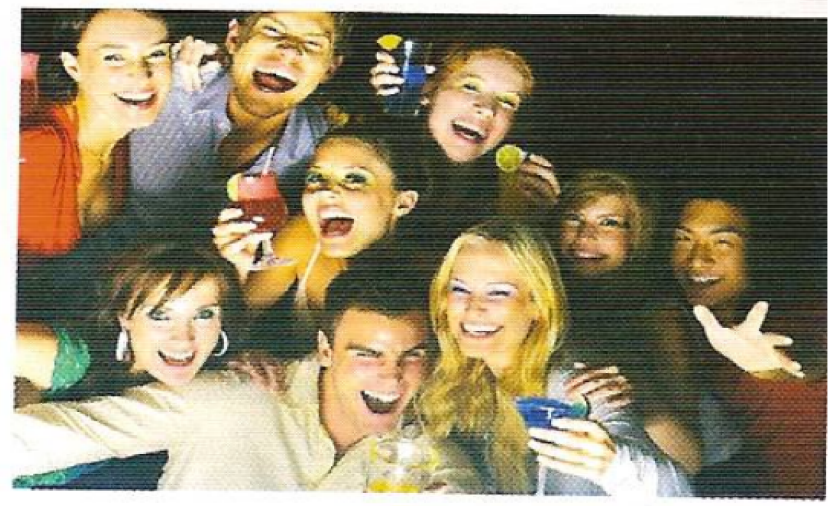

22. Crianças e adolescentes não poderão ingerir álcool sem riscos para a saúde, dado o sistema nervoso ser, nesta fase do crescimento, muito mais sensível ao álcool do que no adulto.

Os manuais escolares, ao apresentarem as consequências individuais a curto e longo prazo, bem como consequências familiares e sociais, de alguma forma podem desenvolver nos alunos a capacidade de prever as consequências das suas decisões. 
Por último, no que respeita ao treino da assertividade, os manuais escolares não apresentam propostas de atividades ou situações que o permitam aos alunos.

\section{Conclusões}

A temática da prevenção do consumo de álcool é abordada pelos sete manuais escolares de Ciências Naturais do $9 .^{\circ}$ ano de escolaridade. No entanto, possuindo estes, em média, 213,4 páginas, apenas dedicam a esta temática uma média de 2,7 páginas.

Constata-se que nenhum manual aborda todos os tópicos sobre alcoolismo referidos na grelha de análise. Verifica-se ainda que os manuais diferem muito no que respeita ao número de conteúdos abordados. O manual que aborda um menor número de conteúdos é o manual escolar M3 e o que aborda um maior número de conteúdos é o manual escolar M2.

A análise de conteúdo revelou que a maioria dos manuais não desenvolve os tópicos com a profundidade que seria desejável. Constata-se, ainda assim, que há conteúdos que, embora não tenham sido explorados em profundidade, foram apresentados em todos os manuais escolares, como sejam: a identificação do álcool como uma droga; as consequências individuais a curto e longo prazo; a distinção entre o consumo de álcool e a dependência (esta foi referida por todos os manuais escolares, à exceção de um). Em oposição, nenhum manual escolar faz a distinção entre bebidas alcoólicas fermentadas e destiladas e apenas um manual escolar compara a graduação de diferentes bebidas alcoólicas. Apenas um manual escolar define, ainda que superficialmente, bebidas alcoólicas, sendo também o único que apresenta a fórmula de cálculo da taxa de alcoolemia no sangue. A desmistificação das crenças sobre o álcool é referida apenas por dois dos manuais escolares e os determinantes do alcoolismo são referidos apenas por um manual escolar.

Pela análise efetuada aos manuais escolares, constata-se que não existe, na maioria deles, uma preocupação em combater as crenças e mitos associados ao consumo de álcool nem em informar sobre os níveis de consumo de alto e baixo risco, e muito menos em envolver o aluno e torná-lo capaz de estabelecer os níveis de consumo aceitáveis e os de risco. Nenhum 
sugere atividades para desenvolver nos alunos competências para resistir às pressões que os levam ao consumo.

Pode ainda concluir-se que as estratégias preventivas sobre 0 consumo de álcool, constantes dos manuais escolares, são sobretudo estratégias informativas e, tal como refere Tovar (1995), apesar de informar ser uma forma de prevenir, o mesmo autor duvida que a informação, só por si, seja capaz de suscitar atitudes pró-abstinência e preventivas. Seria importante, tal como defendem Calafat e Munar (1999), que os manuais escolares contemplassem estratégias baseadas nos valores e tomada de decisões e estratégias baseadas no desenvolvimento de competências sociais, integrando uma componente de maior reflexão e interatividade, pois, de acordo com os mesmos autores, aumentaria a eficácia preventiva.

\section{Implicações}

No que se refere à prevenção do consumo de álcool, é importante que se reforce a abordagem das consequências do consumo de álcool a curto prazo, as consequências familiares e sociais, e que se desmistifiquem as crenças associadas ao consumo de álcool. Dado que o que se pretende é uma mudança de atitudes e comportamentos, segundo Calafat e Munar (1999), dever-se-ão utilizar as técnicas de "estudo de caso, de análise crítica de mensagens publicitárias, de análise e resolução de problemas" (p. 64) e técnicas de treino de competências e comportamentos. Por exemplo, é preciso que as crianças e jovens aprendam a rejeitar o álcool, enfrentando situações de oferta, desenvolvam a assertividade e a capacidade de comunicar, aprendendo a defender a sua opinião, bem como desenvolvam a capacidade de prever as consequências das suas decisões, aprendendo assim a tomar as decisões adequadas (Tovar, 1995).

Segundo Calafat e Munar (1999), qualquer que seja a metodologia e técnicas selecionadas, é imperativo que sejam interativas, promotoras de reflexão, que entendam o aluno como protagonista do processo educativo e o estimulem a partilhar as suas dúvidas e a expor as suas crenças, de modo a que se faça uma análise crítica das mesmas e se apresentem modos alternativos de atuação. Mais se acrescenta que as intervenções específicas, para serem eficazes, devem realizar-se em 10 a 15 sessões, durante um período aproximado de 5 semanas (Calafat \& Munar, 1999). 
Uma vez que os resultados deste estudo revelaram que a proposta de abordagem da problemática do alcoolismo se centra sobretudo na componente informativa, não se preocupando com o desenvolvimento de valores e de competências sociais, é necessário que os mesmos passem a integrar estas componentes. Por esse motivo, os manuais escolares devem apresentar uma oferta mais variada de estratégias com verdadeiro impacte na prevenção do consumo de álcool, ou o Ministério da Educação português deve selecionar, adaptar ou editar um manual sobre prevenção do consumo de álcool que sirva de apoio à implementação, por parte dos professores, de estratégias preventivas. Poder-se-á referir que já existem indicações emanadas do Ministério da Educação, como seja a publicação intitulada Consumo de Substâncias Psicoactivas e Prevenção em Meio Escolar (2007).

Embora muitos professores orientem a sua prática letiva pelo manual escolar, é igualmente imprescindível uma boa formação de professores, já que esta permite maior autonomia dos docentes face a este recurso educativo, tornando-os, muitas vezes, competentes para criar os seus próprios materiais didáticos. Recomenda-se, portanto, formação inicial e contínua de professores no que respeita à educação para a saúde na prevenção dos consumos de drogas legais e ilegais.

\section{Limitações do estudo}

A principal limitação deste estudo é a não inclusão, na amostra, dos manuais escolares adotados recentemente. Um estudo com os manuais escolares atuais será realizado em breve e poderá ser útil na identificação de lacunas presentes no que respeita aos conteúdos e metodologias para a abordagem do tema do alcoolismo. Perante essas eventuais omissões, poderão ser apresentadas sugestões para a melhoria do desempenho na prática docente.

\section{Notas}

1 Este artigo foi elaborado com base na tese de mestrado da primeira autora (Dias, 2008). 


\section{Referências}

Bardin, L. (1995). Análise de conteúdo. Lisboa: Edições 70.

Calafat, A. (2002). Estrategias preventivas del abuso de alcohol. Adicciones, 14(Supl. 1), 317-335.

Calafat, A., \& Munar, M. A. (1999). Actuar es posible: Educación sobre el alcohol. Madrid: Ministerio del Interior.

Chiappetta, E., Fillman, D., \& Sethna, G. (1991). A method to quantify major themes of scientific literacy in science textbooks. Journal of Research in Science Teaching, 28(8), 713-725.

Currie, C., Zanotti, C. Morgan, A., Currie, D. Looze , M., Roberts, ...Barnekow, V. (Eds.). (2012). Social determinants of health and well-being among young people. Health behaviour in school-aged children (HBSC) study: International report from the 2009/2010 survey. Copenhaga: WHO Regional Office for Europe.

De Ketele, J. M., \& Roegiers, X. (1999). Metodologia da recolha de dados. Fundamentos dos métodos de observação, de questionários, de entrevistas e de estudo de documentos. Lisboa: Instituto Piaget.

Dias, A. (2008). Prevenção do consumo de álcool em jovens escolarizados de Cabeceiras de Basto: Um estudo efectuado com professores e com manuais escolares de ciências naturais. Tese de mestrado não publicada. Instituto de Educação da Universidade do Minho, Braga, Portugal.

Gérard, F., \& Roegiers, X. (1998). Conceber e avaliar manuais escolares. Porto: Porto Edito-ra.

Hibell, B., Andersson, B., Bjarnason, T., Kokkevi, A., Morgan, M., \& Narusk, A. (Eds.). (1997). The 1995 ESPAD report: Alcohol and other drug use among students in 26 European countries. Suécia: The Swedish Council for Information on Alcohol and Other Drugs (CAN).

Hibell, B., Andersson, B., Bjarnasson, T., Kokkevi, A., Morgan, M., \& Sabroe, S. (Eds.). (2000). The 1999 ESPAD report: Alcohol and other drug use among students in 30 European countries. Suécia: The Swedish Council for Information on Alcohol and Other Drugs (CAN).

Hibell, B., Andersson, B., Bjarnasson, T., Ahlström, S., Balakireva, O., Kokkevi, A., \& Morgan, M. (Eds.). (2004). The ESPAD report 2003: Alcohol and other drug use among students in 35 European countries. Suécia: The Swedish Council for Information on Alcohol and Other Drugs (CAN).

Hibell, B., Guttormsson, U., Ahlström, S., Balakireva, O., Bjarnason, T., Kokkevi, A., \& Kraus, L. (Eds.). (2009). The 2007 ESPAD report: Substance use among students in 35 European countries. Suécia: The Swedish Council for Information on Alcohol and Other Drugs (CAN).

Hibell, B., Guttormsson, U., Ahlström, S., Balakireva, O., Bjarnason, T., Kokkevi, A., \& Kraus, L. (Eds.). (2012). The 2011 ESPAD report: Substance use among students in 36 European countries. Suécia: The Swedish Council for Information on Alcohol and Other Drugs (CAN).

McKinnon, S., O'Rourke, K., Thompson, S., \& Berumen, J. (2004). Alcohol use and abuse by adolescents: The impact of living in a border community. Journal of Adolescent Health, 34(1), 88-93. 
Ministério da Educação (2001). A rede nacional de escolas promotoras de saúde. Lisboa: Ministério da Saúde.

Ministério da Educação (2007). Consumo de substâncias psicoactivas e prevenção em meio escolar. Lisboa: Editorial do Ministério da Educação.

Nogueira, A., Silva, E., Santos, D., \& Azevedo, M. (2008). Plano de promoção da educação, segurança e prevenção rodoviária de Braga - Conduz a tua vida com segurança. Braga: Instituto de Educação e Psicologia, Universidade do Minho.

Organização Mundial de Saúde (OMS) (2000). Plano europeu de acção sobre o álcool 2000-2005. Porto: Instituto de Alcoologia.

Organização Mundial de Saúde (OMS) (2005). Politique de la région européenne de l'OMS en matière d'alcool: Situation actuelle et perspectives. Copenhaga: Organização Mundial de Saúde.

Organização Mundial de Saúde (OMS) (2007). Comité de expertos de la OMS en problemas relacionados con el consumo de alcohol - $2^{a}$ Reunión 2006. Genebra: Organização Mundial de Saúde

Parcerisa, A. (1997). Materiales curriculares: Como elaborarlos, seleccionarlos y usarlos. Barcelona: Graó.

Pascual, F. (2002). Imagen social de las bebidas alcohólicas. Adicciones, 14(Supl. 1), 115-122.

Pechansky, F., Szobot, C., \& Scivoletto, S. (2004). Uso de álcool entre adolescentes: Conceitos, características epidemiológicas e factores etiopatogénicos. Revista Brasileira de Psiquiatria, 26(Supl. I), 14-17.

Quivy, R., \& Campenhoudt, L. (2003). Manual de investigação em ciências sociais. Lisboa: Gradiva.

Sampaio, D., Baptista, M., Matos, M., \& Silva, M. (2007). Grupo de trabalho de educação sexual/saúde: Relatório final. Lisboa: Ministério da Educação.

Teixeira, F., Couceiro, F., Veiga, L., \& Martins, I. (1999). A educação científica veiculada por manuais escolares de estudo do meio do $1^{\circ} \mathrm{CEB}$, no que respeita à reprodução humana. In V. Trindade et al. (Coords.), Metodologias do ensino das ciências investigação e prática dos professores (pp. 277-288). Évora: Departamento de Pedagogia e Educação, Universidade de Évora.

Tovar, C. (1995). Alcoholismo. In C. Eseverri et al. (Orgs.). Educar para la salud: Drogodependencias (pp. 105-142). Madrid: CCS.

\section{Legislação consultada}

Despacho interno do SEE de 27 de setembro de 2006. Lisboa: Ministério da Educação. Disponível em http://portal.arsnorte.minsaude.pt/portal/page/portal/presse/ Enquadramen-to\%20legal\%20e\%20normativo/10\%20-\%20Despacho\%20\% 2015987\%20de\%2027\%20set_0.pdf (acesso em 12 de dezembro de 2013).

Despacho n. ${ }^{\circ} 29865 / 2007$, de 30 de novembro. Diário da República $n^{\circ} 249-2^{a}$ Série. Lisboa: Ministério da Educação.

Lei n. ${ }^{\circ}$ 47/2006, de 28 de agosto. Diário da República $n^{\circ} 165-1^{a}$ Série. Lisboa: Assembleia da República. 
PREVENTION OF ALCOHOL CONSUMPTION IN PORTUGUESE NATURAL SCIENCES' TEXTBOOKS

\begin{abstract}
Alcohol consumption is a serious problem, very prevalent and growing among schooled adolescents. For that reason, the prevention of alcohol consumption should be carried out at school. School textbooks are a resource to be used in preventive processes. The objective of the present investigation is to analyze the approaches to contents concerning alcohol consumption proposed in 9th grade Portuguese Natural Sciences' school textbooks. For that, one has proceeded to the contents analysis of the seven school books in Portugal for the school year of 2008/2009. These textbooks will be used until 2014. School textbooks approach the alcohol consumption problem in a very incomplete and superficial way. None suggests activities to develop students' competences to resist to pressures leading to consumption. School textbook authors should take into account the need to discuss this topic.
\end{abstract}

Keywords

School textbooks; Alcoholism prevention; Education; Adolescence

PRÉVENTION DE LA CONSOMMATION D'ALCOOL DANS LES MANUELS SCOLAIRES DE SCIENCES NATURELLES PORTUGAISES

\title{
Résumé
}

La consommation d'alcool est très répandue parmi les étudiants adolescents devient, de plus en plus, un problème sérieux. C'est pourquoi la prévention doit se faire à l'école et les manuels scolaires sont une ressource importante dans ce processus. Cette étude analyse les approches à la question de la consommation d'alcool proposées par les manuels portugais de Sciences Naturelles (9ième année de scolarité). Ainsi, nous avons analysé sept manuels scolaires existant au Portugal pour l'année scolaire 2008/2009 et 
utilisés jusqu'à 2014. Ces manuels traitent la question de la consommation d'alcool de façon très incomplète et assez superficielle. En aucun cas, nous avons trouvé des suggestions d'activités qui puissent développer chez les jeunes les compétences nécessaires pour résister aux pressions qui mènent, souvent, à la consommation. Les auteurs de manuels devraient prendre en compte le besoin d'approfondir cette thématique.

Mots-clé

Manuels scolaires; Prévention de l'alcoolisme; Education; Adolescence

Recebido em junho/2014

Aceite para publicação em outubro/2014

i Agrupamento de Escolas de Cabeceiras de Basto, Portugal.

ii Instituto de Educação, Universidade do Minho, Portugal.

Toda a correspondência relativa a este artigo deve ser enviada para: José Alberto Gomes Precioso, Instituto de Educação, Universidade do Minho, Campus de Gualtar, 4710-057 Braga; E-mail: precioso@ie.uminho.pt 